\title{
Empleo de cromitas refractarias para la obtención de fundentes aglomerados utilizados en la soldadura automática por arco sumergido
} $(\mathrm{SAW})^{(\cdot)}$

\author{
L. Perdomo-González*, R. Quintana-Puchol*, A. Cruz-Crespo*, J. Castellanos- \\ Estupiñán ${ }^{* *}$, L.L. García-Sánchez ${ }^{* * *}$, A. Formoso-Prego ${ }^{* * * *}$ y A. Cores-Sánchez ${ }^{* * *}$
}

Resumen En el presente trabajo se obtienen ferrocromo de alto carbono (ferrocromo de carga) y escorias a partir del proceso de reducción de cromitas refractarias. Mediante la utilización de un diseño de experimento de relaciones entre componentes, se estudia la obtención de aleaciones y escorias. La composición química de ambas garantiza su aplicación para la conformación de cargas aleantes y matrices de fundentes aglomerados, para el relleno superficial mediante soldadura automática por arco sumergido (SAW). El proceso de fusión-reducción se realiza en un horno eléctrico de arco.

Palabras clave Fundentes para la soldadura por arco sumergido. Reducción carbotérmica. Ferrocromo. Escoria.

\section{Use of refractory chromites for obtaining fluxes amassed employed in submerged arc welding (SAW)}

\begin{abstract}
In the present work high carbon ferrochrome (load ferrochrome) and slags are obtained, starting from the metallurgic reductive processing of refractory chromites. The obtaining of alloys and slags is studied using an experiment design based in the relationships among components. The chemical compositions of alloys and slags guarantee their application for the conformation of alloys loads and matrix fluxes amassed for the superficial filling by means of submerged arc welding (SAW). The melting-reduction process is carried out in an electric arc furnace.
\end{abstract}

Keywords Fluxes for the submerged arc welding. Carbothermic reduction. Ferrochrome. Slag.

\section{INTRODUCCIÓN}

La práctica de la metalurgia de las ferroaleaciones dirige su atención al empleo de minerales o concentrados de alta ley, con el propósito de aumentar los volúmenes de metal obtenidos al procesar una menor cantidad de mineral, lo que permite reducir los consumos de energía y disminuir los volúmenes de escoria, mejorando considerablemente los indicadores del proceso de obtención. Este aspecto está limitado por la tendencia constante a la disminución de las reservas de minerales de alta ley (espe- cialmente las cromitas), lo que obliga a introducir nuevas tecnologías de beneficio y/o procesamiento metalúrgico.

En la práctica tradicional, el objetivo básico de estos procesos es la obtención de la ferroaleación al cromo, siendo la escoria un producto secundario que no ha encontrado, en la mayoría de los casos, aplicación práctica posterior predeterminada, habiéndose orientado su aplicación a otras industrias consumidoras del residual. Por esta razón, resulta de vital importancia planificar aplicaciones a las escorias procedentes de los procesos metalúrgicos,

(•) Trabajo recibido el día 29 de abril de 2003 y aceptado en su forma final el día 1 de agosto de 2003.

(*) Centro de Investigaciones de Soldadura, Universidad Central de Las Villas, Carretera a Camajuaní km. $5^{1 / 2}$ Santa Clara Villa Clara. CP 54830 (Cuba).

(**) Facultad de Química Farmacia, Universidad Central de Las Villas, Carretera a Camajuaní km. 5 ? Santa Clara Villa Clara, CP 54830 (Cuba).

${ }^{(* * *)}$ Centro de Investigaciones Metalúrgicas (CIME), Avda. 51, 23611 esq. 240, La Lisa, Ciudad de La Habana (Cuba).

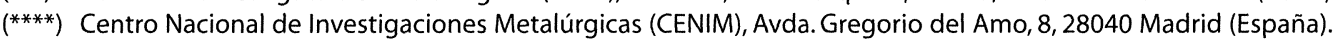


lo cual permite realizar modificaciones en las formulaciones de carga para obtener las aleaciones y escorias con los requerimientos preconcebi$\operatorname{dos}^{[1}$ y 2$]$.

En la conformación de las cargas aleantes de los materiales para la soldadura por arco eléctrico (electrodos y fundentes para Sumerged Meeting Arc Welding (SMAW) y Sumerged Arc Welding (SAW), respectivamente) se emplean, frecuentemente, las ferroaleaciones, que garantizan la composición química de los depósitos de soldadura y la desoxidación, de lo que se derivan las propiedades mecánicas de los mismos ${ }^{[3]}$.

Además de la carga aleante, los materiales para soldar están formados por un soporte matricial constituido fundamentalmente por un sistema de óxidos y sales que garantiza las propiedades tecnológicas y metalúrgicas (escoria-metal del baño) durante el proceso de soldadura. Estos sistemas matriciales se conforman, fundamentalmente, a partir de óxidos puros y/o de concentrados minerales generalmente no metálicos ${ }^{[4}$ y 5$]$, los que de acuerdo a los criterios del fabricante pueden sinterizarse, vitrificarse mediante fusión o emplearse como una mezcla preelaborada en proporciones diferentes establecidas.

Cuba posee reservas apreciables de cromitas que, en su mayoría, son refractarias ${ }^{[6-9]}$. El empleo de estas cromitas para la obtención de ferrocromo tiene la limitación de que las temperaturas de $\mathrm{f}$ usión son muy altas, siendo los volúmenes de ferroaleaciones que producen bajos y los de escorias altos. Si se analizan estas cromitas, no con la óptica de utilizarla como materia prima para obtener solo ferrocromo sino con el objetivo de convertir los productos del proceso de fusión-reducción en componentes esenciales de un fundente aglomerado aleado para la SAW, se alcanzará un empleo más integral y una distribución más equitativa de la energía por producto y, por ende, una disminución sustancial de los costos de producción $^{[1}$ y 2].

El $\mathrm{Al}_{2} \mathrm{O}_{3}, \mathrm{MgO}, \mathrm{CaO}$ y $\mathrm{SiO}_{2}$ son compuestos típicos constituyentes de sistemas de óxidos empleados en la fabricación de matrices de fundentes para la soldadura SAW. El $\mathrm{Cr}_{2} \mathrm{O}_{3}$ y $\mathrm{FeO}$ son compuestos que, en altos contenidos, influyen negativamente en las funciones metalúrgicas de estos sistemas matriciales de óxidos en la soldadura ${ }^{[4]}$; por tanto, si mediante un proceso dado se logra extraer el cromo y el hierro del sistema de óxidos de la cromita (ganga del mineral) se tendrá un sistema de escoria capaz de ser modificado para la confec- ción de las matrices de estos consumibles y una aleación de cromo factible de emplearse en la confección de la carga aleante.

Esta conjugación compleja de objetivos se puede conseguir si se someten las cromitas a un proceso de reducción, mediante una formulación correcta de la carga, que permita realizar un balance para la obtención de un sistema de óxidos que se enmarque en la zona de temperaturas de 1.500 a $1.600{ }^{\circ} \mathrm{C}$ del "campo de cristalización primario" de la espínela del diagrama de fases del sistema $\mathrm{Al}_{2} \mathrm{O}_{3}-\mathrm{SiO}_{2}-\mathrm{MgO}$ (Fig. 1) ${ }^{[10]}$ y de ferrocromo con más del $50 \%$ de cromo y un contenido alto en carburos.

En el marco de colaboración entre centros de investigación de Cuba y España, en el presente trabajo se estudia la utilización de cromitas refractarias para la obtención de ferroaleaciones y escorias con aplicación en la conformación de cargas aleantes y matrices fundentes aglomeradas, empleadas en la soldadura SAW ${ }^{[11-13]}$.

\section{PARTE EXPERIMENTAL}

\subsection{Materias primas}

La composición química de las materias primas utilizadas se ofrece en la tabla I.

Las cromitas refractarias se emplean en la industria siderúrgica cubana en la preparación de materiales refractarios ${ }^{[1}$ y 2$]$. La relación $\mathrm{Cr}_{2} \mathrm{O}_{3} / \mathrm{FeO}$, de 2,26 confirma el carácter refractario, que está por debajo de la relación de la cromita empleada como materia prima para la obtención de ferrocromo (2,5); además el contenido de $\mathrm{Cr}_{2} \mathrm{O}_{3}$ está por debajo del valor recomendado (> $40 \%$ $\mathrm{Cr}_{2} \mathrm{O}_{3}$ ), por lo que los volúmenes de ferroaleación y contenidos de cromo resultaron bajos (31 y $65 \%$, respectivamente). A pesar de estos datos, el potencial teórico de la cromita determinado a partir de la composición química, permite afirmar que se puede considerar como una fuente alternativa para la obtención de aleaciones de cromo. Estas ferroaleaciones se pueden emplear, directamente, en la fabricación de cargas aleantes de materiales para soldar destinados al recargue (electrodos tubulares y fundentes aglomerados), donde las exigencias de calidad (alto contenido de carbono) son diferentes a las de la industria metalúrgica.

Se observa que un $50 \%$ de la composición química de la cromita está formada por $\mathrm{Al}_{2} \mathrm{O}_{3}, \mathrm{MgO}$ y $\mathrm{SiO}_{2}$, en relaciones porcentuales $\left(\mathrm{Al}_{2} \mathrm{O}_{3}: \mathrm{MgO}\right.$ : 
Empleo de cromitas refractarias para la obtención de fundentes aglomerados utilizados en la soldadura automática por arco sumergido (SAW) L. Perdomo-González, R. Quintana-Puchol, A. Cruz-Crespo, J. Castellanos-Estupiñán, L.L. García-Sánchez,

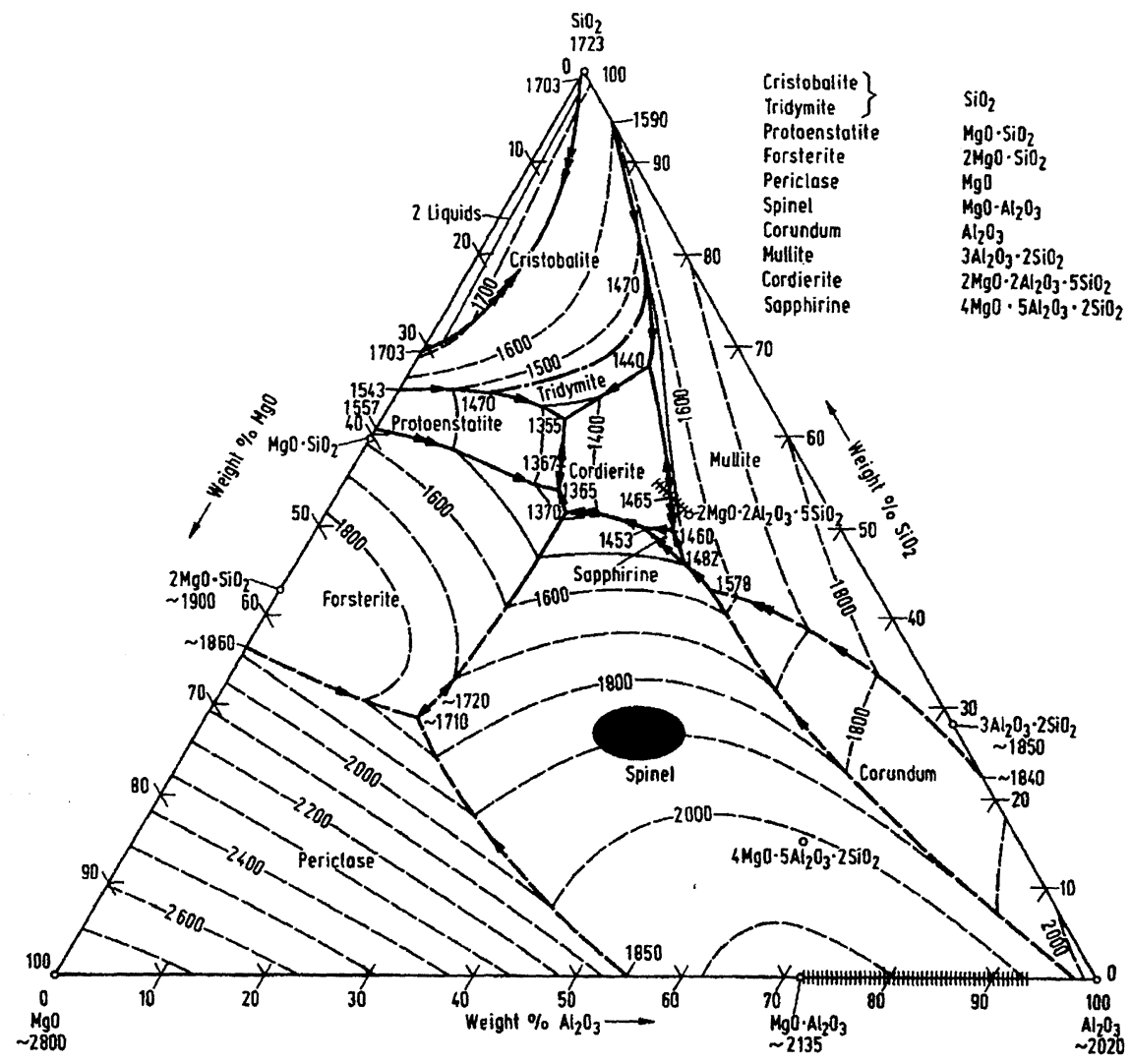

Figura 1. Diagrama de fases $\mathrm{Al}_{2} \mathrm{O}_{3}-\mathrm{MgO}-\mathrm{SiO}_{2}$.

Figure 1. Phases diagram $\mathrm{Al}_{2} \mathrm{O}_{3}-\mathrm{MgO}-\mathrm{SiO}_{2}$.

Tabla I. Composición química de las materias primas y la ceniza del coque, \% en masa

Table I. Chemical composition of raw material and the coke ashes, \% in mass

\begin{tabular}{|c|c|c|c|c|c|c|}
\hline $\begin{array}{l}\text { Compo- } \\
\text { nente }\end{array}$ & Cromita & $\begin{array}{c}\text { Arena } \\
\text { sílice }\end{array}$ & $\begin{array}{l}\text { Roca } \\
\text { caliza }\end{array}$ & Fluorita & Coque & $\begin{array}{c}\text { Ceniza del } \\
\text { coque }\end{array}$ \\
\hline $\mathrm{Cr}_{2} \mathrm{O}_{3}$ & 32,87 & & & & & \\
\hline $\mathrm{SiO}_{2}$ & 5,79 & 90,32 & 0,34 & 3,24 & - & 88,08 \\
\hline $\mathrm{TiO}_{2}$ & & 0,48 & - & - & - & - \\
\hline $\mathrm{Al}_{2} \mathrm{O}_{3}$ & 26,06 & 0,73 & 0,23 & 0,43 & - & - \\
\hline $\mathrm{Fe}_{2} \mathrm{O}_{3}$ & 16,16 & 1,30 & 0,17 & - & - & - \\
\hline $\mathrm{CaO}$ & 0,42 & 1,63 & 55,20 & 0,22 & - & 2,17 \\
\hline $\mathrm{MgO}$ & 16,00 & 0,90 & 0,68 & 0,10 & - & 1,58 \\
\hline $\mathrm{K}_{2} \mathrm{O}$ & & 0,73 & - & - & - & 3,75 \\
\hline $\mathrm{Na}_{2} \mathrm{O}$ & & 0,81 & - & - & - & 4,42 \\
\hline $\mathrm{CaF}_{2}$ & & - & - & 96,1 & - & - \\
\hline$C_{\text {fijo }}$ & & - & - & - & 83,40 & - \\
\hline Cenizas & & & & & 12,00 & \\
\hline$S$ & & - & - & - & 1,10 & - \\
\hline Humedad & & - & - & - & 3,50 & - \\
\hline PPI & & 2,01 & 44,37 & - & - & - \\
\hline
\end{tabular}

$\left.\mathrm{SiO}_{2}=1: 0,6: 0,2\right)$ factibles de adaptarse a las relaciones matriciales de fundente de soldadura $\left\{\mathrm{Al}_{2} \mathrm{O}_{3}: \mathrm{MgO}: \mathrm{SiO}_{2}=1:(0,47-0,77):(0,62-1)\right\}$. Por tanto, al reducirse el $\mathrm{Cr}_{2} \mathrm{O}_{3}$ y el $\mathrm{FeO}$ se obtiene una escoria formada, mayoritariamente, por estos compuestos. Estas relaciones porcentuales permiten ajustar, posteriormente, el sistema de óxidos hasta obtener composiciones químicas que lleguen a representar más del $80 \%$ de la composición de la matriz de un fundente aglomerado. Este aspecto, unido a la posibilidad de empleo de la ferroaleación, hace factible estudiar una tecnología que permita recuperar el cromo y el hierro y adecuar las escorias para su utilización en el desarrollo de fundentes para la SAW.

Como agente reductor se emplea coque que contiene un $12 \%$ de cenizas, en cuya composición prevalece el $\mathrm{SiO}_{2}$ (88\%); el resto de componentes de la ceniza son óxidos de bajo potencial de ionización, lo que favorece la estabilización del arco eléctrico. Como fundentes, se emplean arena sílice $\left(90 \% \mathrm{SiO}_{2}\right)$, caliza $(55 \% \mathrm{CaO})$ y fluorita (96\% $\mathrm{Ca}_{2} \mathrm{~F}$ ). 
Empleo de cromitas refractarias para la obtención de fundentes aglomerados utilizados en la soldadura automática por arco sumergido (SAW) L. Perdomo-González, R. Quintana-Puchol, A. Cruz-Crespo, J. Castellanos-Estupiñán, L.L. García-Sánchez,

A. Formoso-Prego y A. CORES-SÁNCHEZ

\subsection{Análisis por difracción de rayos $\mathrm{X}$ de la cro- mita}

El análisis de fases de la cromita se realiza mediante difracción de rayos X (DRX), utilizando un equipo con radiación monocromática $\mathrm{CuK} \alpha$ filtrada $(\lambda=1,5401 \AA)$ y se realiza el registro con un ángulo de barrido de $2 \theta$ de $10^{\circ}$ a $70^{\circ}$ (Fig. 2).

Las tres fases mineralógicas detectadas son:

- Cromopicotica, (Mg, Fe) (Cr, $\mathrm{Al})_{2} \mathrm{O}_{4}$, de composición calculada, en \%: $38,5 \quad \mathrm{Cr}_{2} \mathrm{O}_{3}$, $12 \mathrm{Al}_{2} \mathrm{O}_{3}$ y $14 \mathrm{FeO}$.

- Antigorita, $\mathrm{Mg}_{6}\left(\mathrm{Si}_{4} \mathrm{O}_{10}\right)(\mathrm{OH})_{8}$, de composición, en \%: $43 \mathrm{MgO}, 41,1 \mathrm{SiO}_{2}$ y $12,9 \mathrm{H}_{2} \mathrm{O}$.

- Clorita, $\left(\mathrm{Mg}_{6-\mathrm{x}-\mathrm{y}} \mathrm{Fe}_{\mathrm{y}} \mathrm{Al}_{\mathrm{x}}\right)\left(\mathrm{Si}_{4-\mathrm{x}} \mathrm{Al}_{\mathrm{x}} \mathrm{O}_{10}\right)(\mathrm{OH})_{8}$. A partir del difractograma la fórmula de la clorita resulta ser, $\left(\mathrm{Mg}_{4,59} \mathrm{Fe}_{0,2} \mathrm{Al}_{1,21}\right)\left(\mathrm{Si}_{2,79} \mathrm{Al}_{1,21} \mathrm{O}_{10}\right)$ $(\mathrm{OH})_{8}$, que expresa la composición química siguiente, en \%: 32,7 $\mathrm{MgO}, 29,8 \mathrm{SiO}_{2}, 22,0$ $\mathrm{Al}_{2} \mathrm{O}_{3}, 2,6 \mathrm{FeO}$ y $12,8 \mathrm{H}_{2} \mathrm{O}$.

Por cálculo, el contenido de cada fase en la cromita es: cromopicotica $85 \%$, antigorita $9 \%$ y clorita $6 \%$. El análisis por DRX permite establecer que la cromita es un mineral muy refractario.

\subsection{Reducción carbotérmica}

La reducción carbotérmica de la cromita por el coque se realizó en un horno eléctrico de arco (HEA) con las siguientes características: crisol de grafito, monofásico, corriente continua, régimen de trabajo $823 \mathrm{~A}$ y $35 \mathrm{~V}$, tiempo de fusión $1 \mathrm{~h}^{[1]}$. Cada uno de los ensayos se realizo por duplicado.

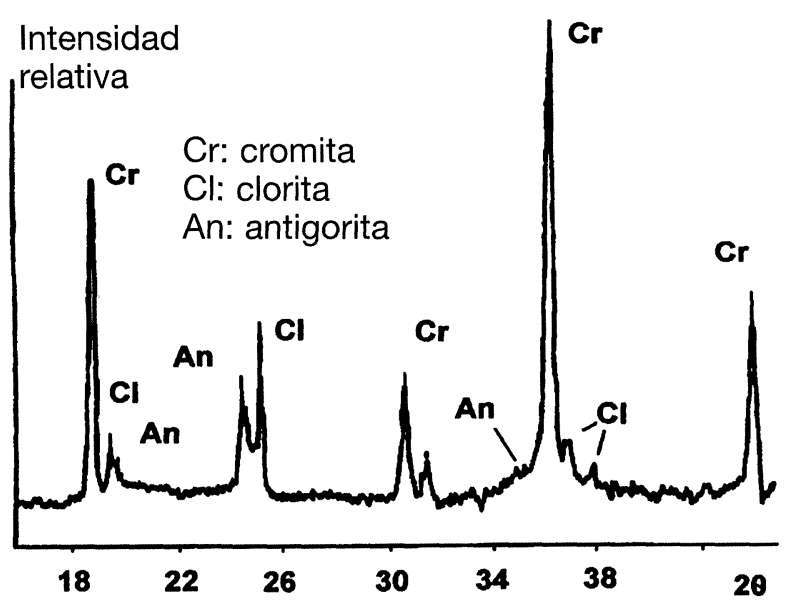

Figura 2. Difractograma de rayos $X$ de la cromita.

Figure 2. Chromite $X$-ray diffractogram.
La energía necesaria para desarrollar el proceso de fusión reducción se suministra por una fuente de corriente del tipo Mansfeld de 1000 A y 48 V. El vertido de la masa fundida se realizó sobre una cubeta con agua a temperatura ambiente. Los parámetros del vertido son los siguientes:

Altura: 0,5-0,6m como máximo.

Temperatura: $1.500-1.600{ }^{\circ} \mathrm{C}$ como mínimo.

Velocidad de vertido: $1-1,5 \mathrm{l} / \mathrm{min}$.

Volumen de agua: $6,6.10^{-2} \mathrm{~m}^{3}$

\subsubsection{Balance de carga al HEA}

El balance de materiales se realiza con el objetivo de conformar una carga para someterla al proceso de fusión-reducción y valorar, a partir de sus resultados, las potencialidades de los productos del proceso, para emplearlos como materias primas básicas en la confección de fundentes para la SAW. Este balance está constituido por dos partes fundamentales: componentes iniciales (carga del horno) y productos finales del proceso ${ }^{[14]}$.

Los componentes iniciales más importantes son: cromita, coque como agente reductor, arena sílice como fundente y oxígeno atmosférico. Los productos obtenidos son: ferrocromo, escoria y productos volátiles formados durante la reducción, principalmente entre el reductor y el oxígeno de la cromita.

El cálculo de los componentes de la carga y de los productos del proceso se rigen por las reacciones siguientes:

$$
\begin{aligned}
& \mathrm{Cr}_{2} \mathrm{O}_{3}+3 \mathrm{C} \rightarrow 2 \mathrm{Cr}+3 \mathrm{CO} \\
& \mathrm{FeO}+\mathrm{C} \rightarrow \mathrm{Fe}+\mathrm{CO} \\
& \mathrm{SiO}+2 \mathrm{C} \rightarrow \mathrm{Si}+2 \mathrm{CO} \\
& 2 \mathrm{C}+\mathrm{O}_{2} \rightarrow 2 \mathrm{CO}
\end{aligned}
$$

De la práctica siderúrgica, se ha tomado que el rendimiento de las reacciones (1) y (2) es como promedio de 90 y $95 \%$, respectivamente ${ }^{[14]}$. La reacción (3) representa la parte de la sílice que puede ser reducida por el carbono y la reacción (4) representa el carbono que reacciona con el oxígeno atmosférico.

El cálculo de la cantidad de carbono se efectúa, considerando un $25 \%$ por encima del estequiométrico y el contenido de sílice se determina, teniendo en cuenta que debe constituir el $30 \%$ del sistema $\mathrm{Al}_{2} \mathrm{O}_{3}-\mathrm{MgO}-\mathrm{SiO}_{2}{ }^{[14]}$.

Los resultados del balance de masa para una base de $1,5 \mathrm{~kg}$ de cromita $\left(32,87 \%\right.$ de $\left.\mathrm{Cr}_{2} \mathrm{O}_{3}\right)$ aparecen en la tabla II. A partir de estos resultados 
Empleo de cromitas refractarias para la obtención de fundentes aglomerados utilizados en la soldadura automática por arco sumergido (SAW) L. Perdomo-González, R. Quintana-Puchol, A. Cruz-Crespo, J. Castellanos-Estupiñán, L.L. García-Sánchez,

A. Formoso-PREgo y A. CORES-SÁNCHEZ

Tabla II. Balance de carga inicial del horno

Table II. Furnace initial load balance

\begin{tabular}{|c|c|c|c|c|}
\hline \multirow{2}{*}{$\begin{array}{l}\text { Material } \\
\text { de entrada }\end{array}$} & \multirow{2}{*}{$\begin{array}{l}\text { química } \\
\text { quición }\end{array}$} & \multicolumn{2}{|c|}{ Cantidad } & \multirow{2}{*}{$\begin{array}{l}\text { Producto } \\
\text { de salida }\end{array}$} \\
\hline & & Entrada, kg & Salida, kg & \\
\hline \multirow{6}{*}{ Cromita $(74,95 \%)$} & $\mathrm{Cr}_{2} \mathrm{O}_{3}$ & 0,515 & 0,052 & \multirow{7}{*}{$\begin{array}{c}\text { Escoria } \\
(50,08 \%)\end{array}$} \\
\hline & $\mathrm{FeO}$ & 0,228 & 0,011 & \\
\hline & $\mathrm{MgO}$ & 0,250 & 0,25 & \\
\hline & $\mathrm{Al}_{2} \mathrm{O}_{3}$ & 0,409 & 0,409 & \\
\hline & $\mathrm{CaO}$ & 0,007 & 0,007 & \\
\hline & $\mathrm{SiO}_{2}$ & 0,091 & 0,068 & \\
\hline Fundente $(10,34 \%)$ & 6) $\mathrm{SiO}_{2}$ & 0,215 & 0,215 & \\
\hline \multirow{2}{*}{ Reductor (11,58 \%) } & $C$ & 0,241 & & \multirow{3}{*}{$\begin{array}{c}\text { Gases } \\
(23,50 \%)\end{array}$} \\
\hline & $\mathrm{CO}$ & & 0,475 & \\
\hline \multirow[t]{5}{*}{ Oxígeno (11,58 \%) } & $\mathrm{O}_{2}$ & 0,065 & & \\
\hline & $\mathrm{Cr}$ & & 0,317 & \multirow{4}{*}{$\begin{array}{c}\text { Ferrocromo } \\
(26,42 \%)\end{array}$} \\
\hline & $\mathrm{Fe}$ & & 0,168 & \\
\hline & $\mathrm{Si}$ & & 0,011 & \\
\hline & $C$ & & 0,038 & \\
\hline $100 \%$ & & 2,021 & 2,021 & $100 \%$ \\
\hline
\end{tabular}

se pudo conformar la carga inicial, integrada de la siguiente manera: cromita $1,5 \mathrm{~kg}$, coque $0,241 \mathrm{~kg}$ y arena sílice $0,215 \mathrm{~kg}$.

En la tabla II se puede observar que, como producto de la fusión-reducción se debería generar un $50,1 \%$ de escoria $(65,46 \%$, respecto a productos sólidos) y un $26,42 \%$ de ferrocromo $(34,54 \%$ con relación a productos sólidos), predominando el volumen de escoria, que constituye un aspecto negativo desde la óptica de obtener solo ferroaleaciones. El hecho de que el proceso permita la obtención de mayor cantidad de escoria que de ferrocromo, no está en contradicción con los objetivos planteados por este trabajo, ya que un fundente está integrado, entre un 70 y $90 \%$, por matriz y el resto corresponde a la carga aleante ${ }^{[5]}$.

La composición química teórica del ferrocromo y la escoria a obtener aparecen en la tabla III.

Se puede apreciar que la carga conformada a partir del balance de masa inicial permite, teóricamente, obtener ferrocromo con un 59,36 \% de cromo y $7,12 \%$ de carbono, que puede emplearse en la conformación de cargas aleantes de fundentes de soldadura destinados al recargue. Además, las escorias que genera el proceso van a estar integradas, básicamente, por un sistema de óxidos del tipo $\mathrm{Al}_{2} \mathrm{O}_{3}-\mathrm{MgO}-\mathrm{SiO}_{2}$ en las relaciones porcentuales siguientes: $\left(\mathrm{Al}_{2} \mathrm{O}_{3}: \mathrm{MgO}: \mathrm{SiO}_{2}=1: 0,56: 0,69\right)$, que
Tabla III. Cálculo teórico de la composición química del ferrocromo y la escoria, \% en masa

Table III. Theoretical calculus of ferrochrome and slag chemical composition, $\%$ in mass

\begin{tabular}{|c|c|c|c|c|c|}
\hline \multicolumn{6}{|c|}{ Escoria } \\
\hline $\mathrm{Cr}_{2} \mathrm{O}_{3}$ & $\mathrm{FeO}$ & $\mathrm{MgO}$ & $\mathrm{Al}_{2} \mathrm{O}_{3}$ & $\mathrm{CaO}$ & $\mathrm{SiO}_{2}$ \\
\hline 5,14 & 1,09 & 24,70 & 40,42 & 0,69 & 27,96 \\
\hline \multicolumn{6}{|c|}{ Ferrocromo } \\
\hline $\mathrm{Cr}$ & & $\mathrm{Fe}$ & $\mathrm{Si}$ & & $C$ \\
\hline 59,36 & & 31,46 & 2,06 & & 7,12 \\
\hline
\end{tabular}

permiten, mediante la adición de nuevos elementos, la obtención de un sistema de óxidos característico de la matriz de un fundente para la SAW.

\subsubsection{Matriz de diseño}

La composición química de la carga, conformada, a partir del balance de masa, establece un sistema de escoria que debe enmarcarse entre las isotermas de 1.830 y $1.860{ }^{\circ} \mathrm{C}$ del diagrama $\mathrm{Al}_{2} \mathrm{O}_{3}-\mathrm{MgO}$. $\mathrm{SiO}_{2}$, indicado en la zona sombreada de la figura 1 . Al tomar en consideración el requisito del contenido de $\mathrm{SiO}_{2}$ en la escoria, para conformar las cargas a partir de cromitas metalúrgicas, se produce, como consecuencia, que los valores de la temperatura de fusión de la carga sean superiores a los valores recomendados para realizar el proceso de fusión-reducción $\left(1.650-1.750^{\circ} \mathrm{C}\right){ }^{[14]}$. Esto, es debido al empleo de cromitas refractarias que presentan temperaturas de fusión muy altas.

Este aspecto obliga a introducir elementos modificadores de la carga o variar las proporciones de los que ya fueron determinados, de forma tal, que permitan reducir la temperatura de fusión.

Para seleccionar los componentes a introducir en la formulación inicial de la carga se han tenido en cuenta dos aspectos fundamentales:

- Que los componentes adicionados disminuyan la temperatura de fusión de la carga (función metalúrgica extractiva).

- Que respondan a la composición química de la matriz de un fundente (requisitos metalúrgicos y tecnológicos del proceso SAW).

La literatura ofrece una amplia gama de fundentes para la soldadura que responden al sistema de óxidos buscado $\left(\mathrm{Al}_{2} \mathrm{O}_{3}-\mathrm{SiO}_{2}-\mathrm{MgO}\right)$, pero no 
todos tienen las mismas relaciones porcentuales entre los óxidos que los conforman y, además, sólo unos pocos tienen en su composición determinados niveles de $\mathrm{Cr}_{2} \mathrm{O}_{3}$, que no afectan al desprendimiento de la escoria del metal depositado y si contribuyen a disminuir las pérdidas de cromo por oxidación, aumentando la transferencia del cromo al baño metálico durante la soldadura ${ }^{[4]}$. Estos niveles de óxido de cromo estarían presentes en estas escorias resultantes de la fusión-reducción.

En la tabla IV se muestra la composición de fundentes comerciales conformados por el sistema de óxidos señalado anteriormente ${ }^{[4]}$.

Tomando en consideración todos estos elementos y los datos de composición de las escorias que debe generar la fusión-reducción, se seleccionaron los siguientes componentes para ser adicionados a la carga del horno: fluorita, caliza y un incremento del contenido de $\mathrm{SiO}_{2}$. Como agente reductor se mantiene el coque.

El estudio del comportamiento metalúrgico de esta carga, a partir de la adición de los nuevos componentes, se realizó utilizando un diseño de experimentos de tipo factorial con relaciones entre componentes. Este modelo se emplea para aquellos casos en que es más interesante conocer la relación entre los componentes de una mezcla que la variación absoluta de los componentes entre si ${ }^{[15}$ y 16$]$.

Los componentes y niveles del diseño se determinan a partir de los resultados del balance de masa inicial y de los rangos de composición química de fundentes comerciales formados por sistemas de óxidos similares en las cantidades siguientes:

$$
\begin{aligned}
& \mathrm{X}_{1}: \text { Fluorita }(0-0,088 \mathrm{~kg} .) \\
& \mathrm{X}_{2}: \text { Arena sílice }(0,236-0,364 \mathrm{~kg} .) \\
& \mathrm{X}_{3} \text { : Coque }(0,231-0,289 \mathrm{~kg} .) \\
& \mathrm{X}_{4} \text { : Caliza }(0-0,094 \mathrm{~kg} .) \\
& \mathrm{X}_{5} \text { : Cromita: } 1,5 \mathrm{~kg} .
\end{aligned}
$$

El mineral de cromita se tomó como base para realizar todas las relaciones, debido a que la es la fuente de cromo y de escoria; por tanto, los elementos que se adicionen a la carga deben permitir su procesamiento, bajar el carácter refractario, aumentar la fluidez, favorecer la reducción de los óxidos metálicos y estabilizar el arco eléctrico.

Las relaciones que se establecen son las siguientes:

$\begin{array}{lll}\text { Relación, } & \text { Nivel mínimo } & \text { Nivel máximo } \\ Z_{1}=X_{1} / X_{5} & 0 & 0,0587 \\ Z_{2}=X_{2} / X_{5} & 0,1587 & 0,2427 \\ Z_{3}=X_{3} / X_{5} & 0,1540 & 0,1927 \\ Z_{4}=X_{4} / X_{5} & 0 & 0,0627\end{array}$

Se establecieron cuatro relaciones, que se toman como variables y se utilizó la ecuación de diseño factorial:

\begin{tabular}{|c|c|c|c|c|c|c|c|}
\hline Tipo & Marca & $\mathrm{SiO}_{2}$ & $\mathrm{Al}_{2} \mathrm{O}_{3}$ & $\mathrm{MgO}$ & $\mathrm{CaO}$ & $\mathrm{CaF}_{2}$ & $\mathrm{MnO}$ \\
\hline \multirow{7}{*}{ Fundido } & $\mathrm{AH}-17 \mathrm{M}$ & $18-22$ & $24-29$ & $8-12$ & $14-18$ & $21-25$ & $<3$ \\
\hline & $\mathrm{AH}-20 \mathrm{C}$ & $19-24$ & $27-32$ & $9-13$ & & $23-25$ & $<0,5$ \\
\hline & FU-22 & 34 & 20 & \multicolumn{2}{|c|}{30} & 15 & 4 \\
\hline & FU-16 & 29 & 19 & \multicolumn{2}{|c|}{27} & 19 & 5 \\
\hline & FU-17 & 26 & 20 & 24 & $<8$ & 15 & - \\
\hline & TAST.11 CrNi & $20-26$ & $26-32$ & $15-20$ & $<8$ & $17-23$ & - \\
\hline & F-302 & $20-26$ & $23-27$ & $16-20$ & $4-8$ & $20-25$ & - \\
\hline \multirow{5}{*}{ Aglomerado } & AHK-57 & 15 & 17 & 25 & 15 & 25 & - \\
\hline & AHK-30 & 12 & 12 & 35 & 15 & 12 & - \\
\hline & SPC-375 & $22-25$ & $23-26$ & $4-6$ & $2-25$ & $19-21$ & - \\
\hline & FC-60 & 20 & 19 & \multicolumn{2}{|r|}{37} & 17 & 4 \\
\hline & FC-40 & 19 & 19 & \multicolumn{2}{|r|}{35} & 25 & - \\
\hline
\end{tabular}

$$
\text { Número de experimentos }=2^{Z}
$$

Tabla IV. Composición química de algunos fundentes comerciales, \% en masa

Table IV. Chemical composition of some commercial fluxes, \% in mass 
Empleo de cromitas refractarias para la obtención de fundentes aglomerados utilizados en la soldadura automática por arco sumergido (SAW) L. Perdomo-González, R. Quintana-Puchol, A. Cruz-Crespo, J. Castellanos-Estupiñán, L.L. García-Sánchez,

A. Formoso-PREGO Y A. CORES-SÁNCHEZ

Cuando $Z=4$, el número de experimentos es 16 .

A partir de la condición de normalidad $\sum_{i=1}^{s} X_{i}=1$ se plantea la relación entre los diferentes componentes de la mezcla de la siguiente forma:

$$
\begin{gathered}
x_{1}+x_{2}+x_{3}+x_{4}+x_{5}=1 \\
Z_{1} x_{5}+z_{2} x_{5}+z_{3} x_{5}+z_{4} x_{5}+x_{5}=1 \\
\left(Z_{1}+Z_{2}+Z_{3}+Z_{4}+1\right) X_{5}=1, \text { donde: } Z_{1}+Z_{2}+ \\
+Z_{3}+Z_{4}+1=\mathrm{R} \\
X_{5}=1 / \mathrm{R}, X_{1}=Z_{1} / \mathrm{R}, X_{2}=Z_{2} / \mathrm{R}, X_{3}=Z_{3} / \mathrm{R} \text { y } X_{4}= \\
Z_{4} / \mathrm{R}
\end{gathered}
$$

La matriz del diseño y las mezclas correspondientes aparecen en la tabla V.

\section{RESULTADOS}

\subsection{Formación de ferroaleaciones}

En la tabla VI se ofrece la composición química de las ferroaleaciones obtenidas.

El contenido de cromo varía entre 50 y $54 \%$ con un valor promedio de $52,12 \%$ de cromo y una desviación estándar (S) de 1,33. Estos parámetros
Tabla VI. Composición química de las ferroaleaciones, \% en masa

Table VI. Chemical composition of ferroalloys, \% in mass

\begin{tabular}{|c|c|c|c|c|c|c|c|c|c|c|}
\hline \multirow{2}{*}{$\begin{array}{c}\mathrm{N}^{\circ} \\
\text { experimento }\end{array}$} & \multicolumn{4}{|c|}{ Matriz del diseño } & \multirow[t]{2}{*}{ R } & \multicolumn{5}{|c|}{ Contenido de mineral } \\
\hline & $\mathrm{Z1}$ & $\mathrm{Z} 2$ & Z3 & Z4 & & $\mathrm{X} 1$ & $\mathrm{X} 2$ & $\mathrm{X} 3$ & $\mathrm{X} 4$ & $X 5$ \\
\hline 1 & - & - & - & - & 1,3127 & 0 & 0,1209 & 0,1173 & 0 & 0,7618 \\
\hline 2 & + & - & - & - & 1,3714 & 0,0428 & 0,1157 & 0,1123 & 0 & 0,7292 \\
\hline 3 & - & + & - & - & 1,3967 & 0 & 0,1738 & 0,1102 & 0 & 0,7160 \\
\hline 4 & + & + & - & - & 1,4554 & 0,0403 & 0,1668 & 0,1058 & 0 & 0,6871 \\
\hline 5 & - & - & + & - & 1,3514 & 0 & 0,1174 & 0,1426 & 0 & 0,7400 \\
\hline 6 & + & - & + & - & 1,4101 & 0,0416 & 0,1125 & 0,1367 & 0 & 0,7092 \\
\hline 7 & - & + & + & - & 1,4354 & 0 & 0,1691 & 0,1342 & 0 & 0,6967 \\
\hline 8 & + & + & + & - & 1,4941 & 0,0393 & 0,1624 & 0,1290 & 0 & 0,6693 \\
\hline 9 & - & - & - & + & 1,3754 & 0 & 0,1154 & 0,1119 & 0,0456 & 0,7271 \\
\hline 10 & + & - & - & + & 1,4341 & 0,0409 & 0,1107 & 0,1074 & 0,0437 & 0,6973 \\
\hline 11 & - & + & - & + & 1,4594 & 0 & 0,1663 & 0,1055 & 0,0430 & 0,6852 \\
\hline 12 & + & + & - & + & 1,5181 & 0,0387 & 0,1599 & 0,1014 & 0,0413 & 0,6587 \\
\hline 13 & - & - & + & + & 1,4141 & 0 & 0,1122 & 0,1363 & 0,0443 & 0,7072 \\
\hline 14 & + & - & + & + & 1,4728 & 0,0399 & 0,1078 & 0,1308 & 0,0426 & 0,6789 \\
\hline 15 & - & + & + & + & 1,4981 & 0 & 0,1620 & 0,1286 & 0,0419 & 0,6675 \\
\hline 16 & + & + & + & + & 1,5568 & 0,0377 & 0,1559 & 0,1238 & 0,0403 & 0,6423 \\
\hline
\end{tabular}

\begin{tabular}{ccccc}
\hline $\mathbf{N}^{\circ}$ & $\mathbf{C r}$ & $\mathbf{C}$ & $\mathrm{Si}$ & $\mathrm{S}$ \\
\hline 1 & 50,08 & 6,01 & 3,09 & 0,010 \\
2 & 51,14 & 5,21 & 2,82 & 0,012 \\
3 & 51,20 & 5,37 & 2,03 & 0,012 \\
4 & 50,92 & 5,75 & 3,40 & 0,010 \\
5 & 50,36 & 5,99 & 2,77 & 0,013 \\
6 & 52,38 & 6,00 & 3,47 & 0,011 \\
7 & 52,57 & 5,00 & 2,58 & 0,011 \\
8 & 52,00 & 6,21 & 3,16 & 0,013 \\
9 & 50,89 & 5,92 & 2,96 & 0,012 \\
10 & 54,22 & 7,55 & 2,73 & 0,013 \\
11 & 54,01 & 5,86 & 2,24 & 0,012 \\
12 & 51,44 & 5,81 & 2,85 & 0,011 \\
13 & 53,98 & 6,16 & 2,01 & 0,012 \\
14 & 53,05 & 6,25 & 3,06 & 0,010 \\
15 & 52,20 & 5,95 & 2,98 & 0,011 \\
16 & 53,40 & 6,32 & 3,01 & 0,010 \\
\hline
\end{tabular}

estadísticos demuestran que los contenidos de cromo en la ferroaleación se enmarcan en rangos

Tabla V. Matriz del diseño de experimentos y mezclas metalúrgicas

Table V. Matrix of the design of experiments and metallurgic mixtures 
relativamente estrechos; esto puede deberse a que la cantidad de cromita en la carga se tomó como referencia fija, lo que establece la relación $\mathrm{Cr}_{2} \mathrm{O}_{3} / \mathrm{FeO}$ para las diferentes mezclas. El contenido de carbono varió entre 5,21 y $7,55 \%$, con un valor promedio de $5,96 \%$ y $S=0,56$. Esta variación puede deberse a la formación de diferentes tipos de carburos de cromo y de hierro, que pueden encontrarse en distintas proporciones dentro de la ferroaleación. En el caso del silicio su contenido en la ferroaleación oscila entre 2,03 y $3,47 \%$, con un valor promedio de $2,82 \%$ y $S=0,43$. Las oscilaciones en los contenidos de silicio pueden deberse a los diferentes grados de solubilidad del silicio en los carburos de hierro y de cromo y a la posible formación de siliciuros. Respecto al azufre sus contenidos oscilan entre 0,010 y $0,013 \%$, presentando un valor promedio de $0,011 \%$ y $S=1,09 \cdot 10^{-3}$. Los niveles de azufre en las diferentes ferroaleaciones son bajos y constantes, lo que no afecta a la calidad para su empleo en la conformación de las cargas aleantes de los materiales para soldar.

Estas ferroaleaciones pueden tener aplicaciones importantes en la confección de cargas aleantes de diferentes fundentes de soldadura y de electrodos tanto sintéticos (D-700) como tubulares destinados al recargue ${ }^{[3]}$.

\subsection{Formación de escorias}

La tabla VII muestra la composición química de las escorias.

Todas se obtienen con contenidos relativa-mente bajos y constantes de $\mathrm{Cr}_{2} \mathrm{O}_{3}$ y $\mathrm{FeO}$, que favorecen el comportamiento como matriz de fundentes. Además, en todas ellas, las relaciones porcentuales de los óxidos del sistema $\mathrm{Al}_{2} \mathrm{O}_{3}-\mathrm{MgO}-\mathrm{SiO}_{2}$ se enmarcan en las relaciones factibles de modificar en una determinada zona del diagrama de fase para la conformación de la matriz de un fundente determinado, lo que garantiza que cualquier escoria pueda ser empleada para estos fines.

Los resultados de las diferentes fusiones, en cuanto a rendimiento de ferrocromo y masa útil, se ofrecen en la tabla VIII.

\subsection{Análisis por DRX de ferroaleaciones y esco- rias}

La caracterización de fases por difracción de rayos $\mathrm{X}$, realizada a ferrocromos que salieron del horno, permite apreciar que están constituidas por $\mathrm{FeCr}$ y $\mathrm{Cr}_{7} \mathrm{C}_{3}$. Se distingue, como fase importante, $\mathrm{Fe}_{3} \mathrm{C}$ (cementita). También se observan pequeñas cantidades de siliciuros de cromo $\left(\mathrm{CrSi}_{2}\right)$ y de hierro $\left(\mathrm{FeSi}_{2}\right.$ y $\left.\mathrm{Fe}_{5} \mathrm{Si}_{3}\right)$.

Tabla VII. Composición química de las escorias, \% en masa

Table VII. Chemical composition of slags, \% in mass

\begin{tabular}{cccccccc}
\hline $\mathbf{N}^{\circ}$ & $\mathrm{SiO}_{2}$ & $\mathrm{Al}_{2} \mathrm{O}_{3}$ & $\mathrm{MgO}$ & $\mathrm{CaO}$ & $\mathrm{CaF}_{2}$ & $\mathrm{Cr}_{2} \mathrm{O}_{3}$ & $\mathrm{FeO}$ \\
\hline 1 & 30,08 & 37,21 & 22,40 & 0,53 & - & 3,01 & 1,22 \\
2 & 27,71 & 35,10 & 21,03 & 0,52 & 6,09 & 2,98 & 1,20 \\
3 & 36,81 & 33,43 & 20,41 & 0,52 & - & 3,04 & 1,21 \\
4 & 34,92 & 31,08 & 19,01 & 0,61 & 6,01 & 2,85 & 1,17 \\
5 & 31,02 & 37,22 & 22,06 & 0,51 & - & 2,98 & 1,06 \\
6 & 28,83 & 34,06 & 20,54 & 0,49 & 6,72 & 2,72 & 1,11 \\
7 & 36,17 & 33,27 & 20,03 & 0,50 & - & 2,60 & 1,05 \\
8 & 34,17 & 31,18 & 18,03 & 0,57 & 5,99 & 3,01 & 1,14 \\
9 & 28,95 & 35,22 & 21,16 & 5,01 & - & 2,58 & 1,21 \\
10 & 26,83 & 33,05 & 20,72 & 4,50 & 6,05 & 2,61 & 1,04 \\
11 & 35,21 & 32,82 & 19,43 & 4,42 & - & 2,80 & 1,12 \\
12 & 32,12 & 30,64 & 19,06 & 5,03 & 5,90 & 2,52 & 1,23 \\
13 & 29,77 & 34,86 & 21,31 & 4,98 & - & 2,25 & 1,14 \\
14 & 27,19 & 33,28 & 19,19 & 5,80 & 6,01 & 2,41 & 1,22 \\
15 & 35,22 & 32,54 & 18,85 & 5,00 & - & 2,27 & 1,07 \\
16 & 30,66 & 30,58 & 19,81 & 5,70 & 5,98 & 2,30 & 1,10 \\
\hline
\end{tabular}


Empleo de cromitas refractarias para la obtención de fundentes aglomerados utilizados en la soldadura automática por arco sumergido (SAW) L. Perdomo-González, R. Quintana-Puchol, A. Cruz-Crespo, J. Castellanos-Estupiñán, L.L. García-Sánchez,

A. ForMOSO-PREGO Y A. CORES-SÁNCHEZ

Tabla VIII. Rendimiento de ferrocromo y masa de escoria útil obtenidos para los diferentes puntos del diseño de experimentos

Table VIII. Yield of ferrochrome and mass of useful slag obtained for the different points of the design of experiments

\begin{tabular}{|c|c|c|c|c|c|c|}
\hline \multirow{3}{*}{$\mathbf{N}^{\circ}$} & \multicolumn{3}{|c|}{$\mathrm{FeCr}$} & \multicolumn{3}{|c|}{ Escoria } \\
\hline & $\begin{array}{c}\text { Masa teórica, } \\
\text { kg }\end{array}$ & $\begin{array}{c}\text { Rendimiento, } \\
\% \\
\end{array}$ & $\begin{array}{c}\text { Desviación } \\
\text { Estándar }\end{array}$ & $\begin{array}{c}\text { Masa teórica, } \\
\text { kg }\end{array}$ & $\begin{array}{c}\text { Masa escoria } \\
\text { útil, kg }\end{array}$ & $\begin{array}{c}\text { Desviación } \\
\text { Estándar }\end{array}$ \\
\hline & & $\left(Y_{1}\right)$ & $S$ & & $\left(Y_{2}\right)$ & $S$ \\
\hline 1 & 0,534 & 49,81 & 3,97 & 1,094 & 0,101 & 0,028 \\
\hline 2 & 0,534 & 57,68 & 5,30 & 1,182 & 0,601 & 0,047 \\
\hline 3 & 0,534 & 50,00 & 4,50 & 1,220 & 0,199 & 0,027 \\
\hline 4 & 0,534 & 62,73 & 5,83 & 1,308 & 0,821 & 0,048 \\
\hline 5 & 0,534 & 60,12 & 4,00 & 1,094 & 0,121 & 0,039 \\
\hline 6 & 0,534 & 73,03 & 3,66 & 1,182 & 0,654 & 0,037 \\
\hline 7 & 0,534 & 63,86 & 6,24 & 1,220 & 0,243 & 0,037 \\
\hline 8 & 0,534 & 73,60 & 4,77 & 1,308 & 0,845 & 0,055 \\
\hline 9 & 0,534 & 53,37 & 3,97 & 1,188 & 0,160 & 0,036 \\
\hline 10 & 0,534 & 66,48 & 4,24 & 1,276 & 0,770 & 0,044 \\
\hline 11 & 0,534 & 54,61 & 3,01 & 1,314 & 0,276 & 0,044 \\
\hline 12 & 0,534 & 72,85 & 5,83 & 1,402 & 1,018 & 0,072 \\
\hline 13 & 0,534 & 67,78 & 4,23 & 1,188 & 0,168 & 0,040 \\
\hline 14 & 0,534 & 74,91 & 5,02 & 1,276 & 0,786 & 0,041 \\
\hline 15 & 0,534 & 72,66 & 5,50 & 1,314 & 0,295 & 0,047 \\
\hline 16 & 0,534 & 76,59 & 6,10 & 1,402 & 1,027 & 0,069 \\
\hline
\end{tabular}

En la aleación metálica que quedó en el horno se observan las mismas fases anteriores pero con contenidos relativamente diferentes; por ejemplo, el contenido de la fase $\mathrm{Fe}_{3} \mathrm{Cr}$ es menor, los contenidos de carburos de cromo y de hierro son superiores; no obstante se aprecian niveles de siliciuros constantes.

Esta determinación permite corroborar que se obtuvieron aleaciones metálicas con contenidos apreciables de ferrocromo y carburos de cromo, fundamentalmente, del tipo $\mathrm{Cr}_{7} \mathrm{C}_{3}$. La presencia de altos contenidos de carburos en el ferrocromo es muy importante para la confección de cargas aleantes de materiales para soldar, destinados al recargue ya que la transferencia del metal al deposito de soldadura se realiza, fundamentalmente, mediante carburos.

El difractograma realizado a las escorias que salieron del horno, presenta una amplia banda que barre desde los $18^{\circ}$ hasta los $38^{\circ}(2 \theta)$ y una pequeña banda entre $\operatorname{los} 42^{\circ}$ y $44^{\circ}(2 \theta)$, lo que demuestra la presencia de una sustancia de bajo ordenamiento cristalino a la difracción de rayos $\mathrm{X}$ y que la posible presencia de ferrocromo en la escoria está por debajo de los límites de detección del método.

\section{DISCUSIÓN}

Al evaluar el comportamiento tecnológico de las diferentes mezclas durante el proceso de fusiónreducción se clasificó en cuatro grupos (Tabla V):

- Muy estables: En este grupo se ubicaron las mezclas que contenían los niveles superiores de fluorita y caliza (mezclas 10, 12, 14 y 16).

- Estables: Las mezclas que contenían los máximos niveles de fluorita y no contenían caliza (2, 4, 6 y 8$)$.

- Medianamente estables: A este comportamiento respondieron las mezclas que no contenían fluorita, con niveles altos de caliza $(9,11,13$ y 15$)$.

- Inestables: En este grupo se ubicaron todas las mezclas que no contenían fluorita, ni calcita $(1,3,5$ y 7$)$.

En la estabilidad, durante la fusión-reducción de la cromita refractaria, jugó un papel destacado la fluorita, debido a su efecto fundente y a la gran fluidez que confiere a la masa fundida.

Al obtenerse ferroaleaciones con composiciones químicas en rangos aplicables al desarrollo de cargas aleantes de fundentes y escorias con 
composiciones diferentes entre los distintos puntos del diseño, pero todas ellas ajustables a la composición de la matriz de un fundente, se decidió no tomar estos parámetros para procesar el diseño y escoger como variables dependientes otros factores que puedan influir directamente en la conformación de un fundente aglomerado aleado como, por ejemplo, el rendimiento de los productos. Por tanto, para procesar el diseño de experimentos se seleccionaron como variables respuestas las siguientes:

- $Y_{1}$ : Rendimiento de ferrocromo (masa de ferrocromo que se obtiene con relación al valor dado por el balance de masa).

- $Y_{2}$ : Masa de escoria útil (masa de escoria que sale del horno cuando se realiza el vertido).

Se escogieron estos dos parámetros principales (variables respuesta) para evaluar el diseño, ya que un fundente aglomerado aleado está integrado básicamente por matriz y carga aleante, por tanto, en la medida en que sea mayor la cantidad de ferroaleación y escoria que se obtenga mayor será, también, la cantidad de fundente que se puede producir. No se incluyó como variable respuesta un parámetro de calidad debido a que todas las ferroaleaciones y escorias son, potencialmente, aplicables al desarrollo de las formulaciones posteriores.

Para la variable $Y_{1}$ (rendimiento de ferrocromo) se obtuvo el siguiente modelo:

$$
\begin{gathered}
Y_{1}=180,818 Z_{1}+30,810 Z_{2}+288,128 Z_{3}+95,209 Z_{4}, \\
R^{2} \text { ajustado: } 0,99537
\end{gathered}
$$

El modelo obtenido es adecuado de acuerdo con la prueba de Fischer. Se puede observar que el ajuste del modelo es muy bueno; las componentes más significativas son $Z_{3}$ (relación coque-cromita), $Z_{1}$ (fluorita-cromita) y, en menor medida, la relación $Z_{4}$ (caliza-cromita). En el primer caso, la influencia sobre el rendimiento de ferrocromo se debe a que el coque es el elemento que reduce los óxidos de hierro y cromo presentes en la cromita, permitiendo la formación de la ferroaleación; además, una cantidad de carbono pasa a la aleación en forma de carburos. La relación fluorita-cromita influye en el rendimiento debido a que la fluorita aumenta considerablemente la fluidez de la escoria, facilitando la reducción del cromo y el hierro y la relación $Z_{4}$ (caliza-cromita) influye de forma positiva ya que la caliza es un fundente que incorpora elementos de bajo potencial de ionización $(6,16 \mathrm{eV})$, lo que favorece el desarrollo del proceso.
Para la variable $\mathrm{Y}_{2}$ (masa de escoria útil) se obtuvo el siguiente modelo:

$$
\begin{gathered}
Y_{2}=10,440 Z_{1}+1,623 Z_{2}-1,020 Z_{3}+1,706 Z_{4} \\
R^{2} \text { ajustado: } 0,98743
\end{gathered}
$$

El modelo resultó adecuado a la prueba de Fischer. El ajuste del modelo es muy bueno. Las relaciones $Z_{1}$ (fluorita-cromita), $Z_{2}$ (arena-cromita) y $\mathrm{Z}_{4}$ (caliza-cromita) influyen positivamente sobre la masa de escoria útil. La relación $Z_{1}$ facilita la reducción de los óxidos de hierro y cromo, disminuyendo el carácter refractario de la escoria y aumentando la fluidez de la misma, lo que permite incrementar la cantidad de escoria que sale del horno. En el caso de la relación $\mathrm{Z}_{2}$, la arena sílice disminuye la temperatura de fusión de la mezcla; sin embargo, aumenta la viscosidad de la mezcla. Su efecto positivo está en correspondencia a su contribución cuantitativa en la mezcla. La relación $\mathrm{Z}_{4}$ influye, fundamentalmente, en la estabilidad del horno y su efecto fundente. En cuanto a la relación $Z_{3}$, resulta negativa debido a que el coque reduce los óxidos de cromo y hierro disminuyendo, por tanto, los volúmenes de escoria que se producen.

$\mathrm{Al}$ analizar los datos de la tabla VIII se observa que los mejores resultados, tanto para el ferrocromo como para la escoria, se obtienen en el punto extremo, 16, del diseño de experimentos; además, en la tabla VI se observa que cualquiera de las ferroaleaciones puede emplearse para conformar la carga aleante de los materiales para soldar (fundentes para el recargue) y, en la tabla VII, se aprecia que la composición de las escorias del punto 16 es ajustable a la composición de las matrices de fundentes para la SAW (Tabla IV). Todo esto conduce a seleccionar esta mezcla como la de mejores resultados, de acuerdo a los objetivos trazados por este trabajo.

A partir de los resultados del diseño de experimentos de relaciones entre componentes, se logró conformar una carga para ser sometida al proceso de reducción integrada de la siguiente manera:

Cromita: 64,2\%; Arena: 15,6\%; Coque: 12,4\%; Caliza: 4\%; Fluorita: 3,8\%

\section{CONCLUSIONES}

- La composición química y las fases mineralógicas de las cromitas refractarias permiten la conformación de cargas metalúrgicas capaces 
Empleo de cromitas refractarias para la obtención de fundentes aglomerados utilizados en la soldadura automática por arco sumergido (SAW) L. Perdomo-González, R. Quintana-Puchol, A. Cruz-Crespo, J. Castellanos-Estupiñán, L.L. García-Sánchez,

A. Formoso-PREGO Y A. CORES-SÁNCHEZ

de generar ferrocromos y escorias con los requisitos de composición necesarios para su empleo en la confección de fundentes aglomerados para el proceso SAW.

- El empleo de un diseño de experimentos de relaciones entre componentes permitió estudiar la conformación de diferentes cargas metalúrgicas estableciéndose la más adecuada a las condiciones de las materias primas y a las demandas de los constituyentes fundamentales de los fundentes para la SAW (Cromita: 64,2\%; Arena: 15,6 \%; Coque: 12,4 \%; Caliza: $4 \%$; Fluorita: $3,8 \%$ ).

- La carga conformada garantiza la obtención, mediante reducción carbotérmica, de ferroaleaciones de cromo con contenidos de 50-54 \% de cromo y $5-7 \%$ de carbono, así como escorias formadas por sistemas de óxidos del tipo $\mathrm{Al}_{2} \mathrm{O}_{3}-\mathrm{MgO}-\mathrm{SiO}_{2}$, modificado por $\mathrm{CaO}$ y $\mathrm{CaF}_{2}$, aplicables al desarrollo de cargas aleantes y matrices de fundentes para el recargue por SAW.

- El estudio por difracción, realizado a las ferroaleaciones obtenidas, indica que las fases predominantes consisten en $\mathrm{FeCr}, \mathrm{Cr}_{7} \mathrm{C}_{3}, \mathrm{Fe}_{3} \mathrm{C}$ y, en menor medida, siliciuros de cromo y hierro, que constituyen fuentes valiosas para la conformación de cargas aleantes de consumibles de soldadura.

\section{REFERENCIAS}

[1] L. Perdomo, Tesis Doctoral, Instituto Superior Minero Metalúrgico de Moa, Cuba, 1999, p. 164.

[2] R. Quintana, L. Perdomo, M. Rodríguez, C. Gómez, A. Cruz, G. Castellanos y O. Pérez, Procedimiento de obtención simultánea de escoria esponjosa y aleaciones de cromo destinadas a la confección de fundentes aglomerados para la soldadura Automática bajo fundente (SAW), Patente No. C22C 33/00, C22C 19/05.

[3] A. Herrera, R. Quintana y A. Duffus, Fundente aleado para el relleno superficial. Memorias, II Encuentro de Ingeniería de Materiales, La Habana 1998, pp. 173-178.

[4] V. Podgayeskil y G. KuzMienKo, Teoría de las escorias, Kiev, Ed. Naukova Dumka, 1988, p. 255.

[5] R. Quintana, G. Castellanos y Z. Rodríguez, Rev. Minería Geología. 15 (1998) 70-74.

[6] E. Leyva, O. Figueredo, A. Guerra, J. Ortíz y J. RodríGuez, Rev. Minería Geología 14 (1997) 69-71.

[7] J. Proenza, Tesis Doctoral, Facultad de Geología, Universidad de Barcelona, Barcelona, 1997.

[8] G. Jiménez, P. Torralba, A. Arrate, L. García, A. CORES y M. FERnÁNDEZ Rev. Metal. Madrid. 27 Madrid (1991)75-84.

[9] G. Jiménez, L. García, A. Formoso, C. Cubillo, A. IsIDRO y A. Cores, Rev. Metal. Madrid 30 (1994) 38-43.

[10] Slag Atlas, Ed. VDEh, Dusseldorf, Germany, 1995, p. 616.

[11] G. JimÉnez, E. SOler, A. Formoso y A. CORES, Rev. Metal. Madrid 28 (1992) 111-118.

[12] R. Quintana, A. Cruz, L. Perdomo, G. Castellanos, L.L. García, A. Formoso y A. Cores, Rev. Metal. Madrid 39 (2003) 25-34.

[13] A. Cruz, R. Quintana, L. Perdomo, L.L. García, A. Formoso y A. Cores, Rev. Metal. Madrid 39 (2003) (114-122).

[14] A. Riss, Production of ferroalloys, Ed. Foreign languages publishing house, Moscow, 1975.

[15] S. Aknazarova y V. Kafarov, Experimental optimization in chemistry and chemical engineering, Ed. Mir, Moscow, 1982.

[16] M. Martínez, W. Suwardjo, L. García, A. Formoso y A. COREs, Rev. Metal. Madrid 38 (2002) 173-182. 